\title{
Aussagekraft und Limitation des Röntgenbildes bei Früh- und Neugeborenen
}

Verbesserte Pränataldiagnostik und die Fortschritte der Neonatologie gehen mit einer wachsenden Zahl von Frühgeborenen und Kindern mit komplizierten Fehlbildungen einher. In Deutschland werden 8-9\% der Kinder zu früh, d. h. nach Definition der WHO vor Ende der 37. SSW geboren, davon überleben mehr als $90 \%$.
In der apparativen Diagnostik zur Therapie kardiorespiratorischer Erkrankungen und Beurteilung von Fehlbildungen kommt bei diesen Kindern neben der Sonografie der konventionellen ThoraxAufnahme eine besondere Bedeutung zu. Je nach Fragestellung werden bei intensivpflichtigen Patienten Aufnahmen des Tho- rax oft um eine Aufnahme des Abdomens erweitert.

Generell müssen alle Röntgenaufnahmen bei Kindern mit strenger rechtfertigender Indikationsstellung und unter Beachtung des größtmöglichen Strahlenschutzes erfolgen. Hier kommt das ALARA-Prinzip (as low as reasonably achieva- 
ble) vordringlich zur Anwendung. In den Leitlinien der Bundesärztekammer sind ärztliche und aufnahmetechnische Qualitätsanforderungen für Röntgenuntersuchungen bei Kindern festgelegt. Zu beachten sind auch die aktualisierten Referenzwerte des Bundesamtes für Strahlenschutz für diagnostische Röntgenanwendungen. Voraussetzung für die Interpretation pathologischer Befunde ist die Kenntnis des Normalbefundes und seiner Varianten.

Patienten neonatologischer Intensivstationen sind meist nicht transportfähig und müssen im Inkubator untersucht werden. Dadurch entstehende Artefakte und eine Vielzahl von Fremdmaterialien wie Katheter und Elektroden können die Beurteilung erschweren. Ein enger Kontakt zum anfordernden Neonatologen und eine eingehende Kenntnis der Klinik und des Spektrums pädiatrischer Krankheitsbilder sind für eine korrekte Befundung essenziell.

Die häufigste Indikation für die Anfertigung von Thorax-Aufnahmen bei Neugeborenen ist die Abklärung einer respiratorischen Störung. Pulmonale Erkrankungen bei Neugeborenen sind vielfältig und in ihrer bildmorphologischen Darstellung nicht immer einfach zu unterscheiden. Je nach Fragestellung, zum Beispiel bei Pleuraerguss oder Fehlbildungen, kommt additiv die Sonografie zur Anwendung, die CT und die MRT bleiben speziellen Indikationen vorbehalten. Im Folgenden werden die Bedeutung und die Grenzen der konventionellen Thorax-Diagnostik bei der Abklärung dieser Erkrankungen anhand wichtiger neonatologischer Krankheitsbilder vorgestellt.

Ein sinnvoller erster Schritt in der Interpretation auffälliger Thorax-Aufnahmen ist die Unterscheidung, ob es sich um ein reifes Neugeborenes oder ein Frühgeborenes handelt und ob eine fokale oder multifokale bzw. generalisierte Lungenerkrankung vorliegt.

Die häufigste generalisierte Lungenerkrankung des Frühgeborenen ist das Atemnotsyndrom (ANS), Synonym: Idiopathic respiratory distress syndrome (IRDS) ( $\triangleright$ Abb. 1). Ursache ist ein Surfactant-Mangel durch Enzymunreife. Es kommt zu einem Kollaps kleinerer und einer Überblähung größerer Alveolen. In den terminalen Bronchiolen können sich

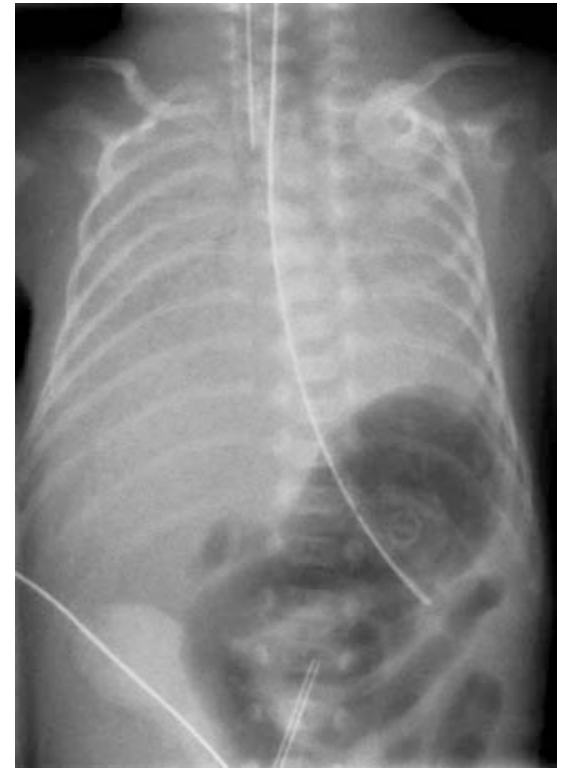

- Abb. 1 Frühgeborenes der 28. SSW am ersten Lebenstag mit Atemnotsyndrom Grad 4. Homogene Verschattung beidseits durch fehlende Belüftung, „weiße Lunge“. Regelrechte Lage eines Trachealtubus und einer Magensonde. Am unteren Bildrand Anschnitt eines Nabelarterienkatheters in tiefer Position.

durch Plasmaexsudationen und Fibrin hyaline Membranen ausbilden. Im Röntgenbild wird das ANS vor der Gabe von Surfactant in 4 Schweregrade eingeteilt:

- Grad 1: retikulogranuläre Zeichnungsvermehrung beidseits

- Grad 2: zusätzlich zu dieser Zeichnungsvermehrung Nachweis von Bronchopneumogrammen mit Ausdehnung über die Herzgrenzen hinaus

- Grad 3: schleierartige bilaterale Eintrübung, fehlende Abgrenzbarkeit von Herz- und Zwerchfellgrenzen

- Grad 4: durch fehlende Belüftung weiße Lunge

In der Versorgung von Frühgeborenen setzt sich zunehmend die Gabe von Surfactant bereits im Kreißsaal vor der Anfertigung einer Röntgenaufnahme durch, in diesen Fällen gelingt diese Einteilung nicht mehr.

Generalisierte Lungenerkrankungen, die Frühgeborene und Reifgeborene betreffen, sind Erkrankungen des alveolaren Wachstums, die prä- oder postnatal durch Schädigung des Lungengewebes auftreten können. Beispielhaft seien hier die chronische Lungenerkrankung des Frühgebore-

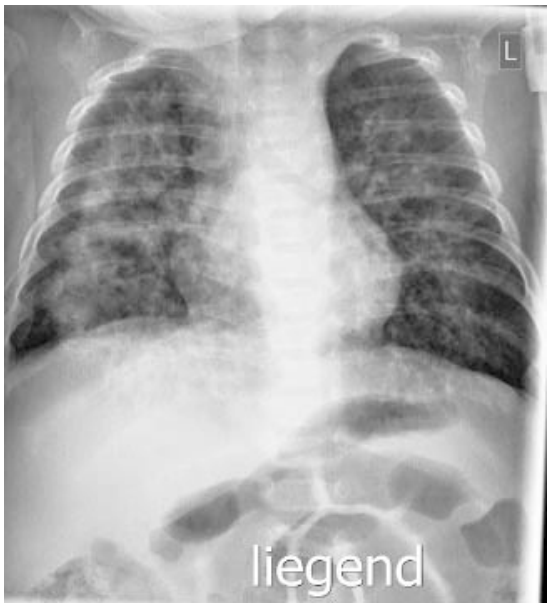

Abb. 2 Ehemaliges Frühgeborenes der 28. SSW im Alter von zwei Monaten mit einer bronchopulmonalen Dysplasie. Beidseits schmetterlingsförmig angeordnete interstitielle Verdichtungen, Überblähung der Peripherie.

nen, die bronchopulmonale Dysplasie (BPD) und die sekundäre pulmonale Dysplasie, z. B. durch ein Oligohydramnion, genannt ( $\triangleright$ Abb. 2). Die Diagnose einer BPD definierte sich ursprünglich klinisch durch die Notwendigkeit einer supplementären Sauerstoffgabe für mindestens 28 Tage bei einem Kind mit einem Gestationsalter von weniger als 32 Wochen. Eine heute gebräuchliche Klassifikation des Institute of Child Health and Human Development unterscheidet verschiedene Schweregrade der BPD und berücksichtigt auch Kinder mit einem Gestationsalter von mehr als 32 Wochen. Pathogenetisch spielen neben anderen Faktoren vor allem eine Überdruckbeatmung und eine Sauerstoffgabe eine Rolle, die von Northway 1967 beschriebenen 4 radiologisch differenzierbaren Stadien werden in Folge verbesserter Therapie in dieser Form meist nicht mehr durchlaufen. Auf lange Sicht kommt es aber dennoch zu chronischen pulmonalen Veränderungen durch die gestörte Entwicklung von Alveolen und pulmonalen Gefäßen.

Differenzialdiagnostisch müssen bei einer diffusen Zeichnungsvermehrung mit pulmonaler Hyperämie und Kardiomegalie ein Vitium cordis oder ein offener Ductus arteriosus Botalli (PDA) echokardiografisch ausgeschlossen werden.

Beim reifen Neugeborenen sind eine pulmonale Retention von Fruchtwasser 




- Abb. 3 Reifes Neugeborenes am ersten Lebenstag mit Mekoniumaspiration. Beidseits fleckförmige Infiltrate, Überblähung der Peripherie. Trachealtubus in zu tiefer Position, Nabelvenenkatheter in zu hoher Lage. Regelrecht liegende Magensonde.

(transitorische Tachypnoe des Neugeborenen, TTN) oder eine Mekoniumaspiration Ausdruck einer generalisierten Lungenerkrankung ( $\triangleright$ Abb. 3). Die TTN imponiert durch eine vermehrte interstitielle Lungenzeichnung und Demarkierung interlobärer Septen, das Lungenvolumen ist normal oder vermehrt. Bei der Mekoniumaspiration kommt es zu einer chemischen Pneumonitis und Inaktivierung von Surfactant. Komplikationen sind eine bakterielle Pneumonie, eine persistierende pulmonale Hypertonie (PPH) oder die Entwicklung eines Pneumothorax.

Im klinischen Alltag weniger häufig sind bei Reifgeborenen Entwicklungsstörungen, wie eine kongenitale azinäre oder alveoläre Dysplasie oder eine kongenitale Störung der Surfactant-Funktion. Auch die kongenitale pulmonale Lymphangiektasie ist selten, hierbei kommt es zu einem Chylothorax. Meist sind Patienten mit Trisomie 21 oder einem Noonan-Syndrom betroffen, auch bei angeborenen Herzfehlern wird sie beobachtet.

Fokale neonatale Lungenerkrankungen zeigen oft bildmorphologisch spezifischere Befunde. Hierzu gehören das kongenitale lobäre Emphysem, die zystische adematoide Malformation der Lunge (CCAM), die pulmonale Sequestration und die einseitige Lungenagenesie ( $\boldsymbol{A}$ Abb. 4 ). Beim

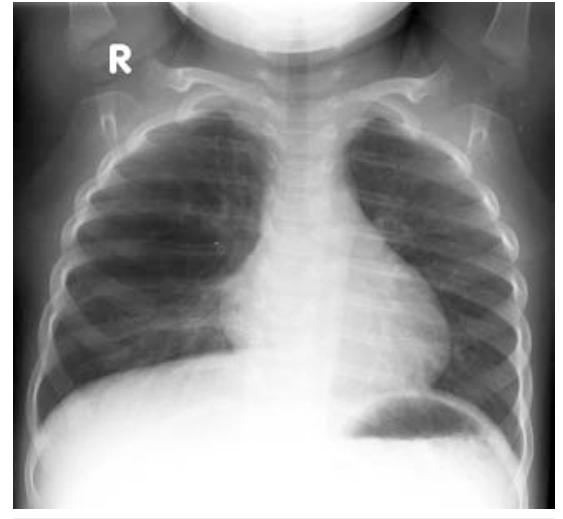

- Abb. 4 Säugling im Alter von 18 Monaten mit einem kongenitalen lobären Emphysem des rechten Oberlappens. Zystoid imponierende, raumfordernde Aufhellung des rechten Oberlappens.

kongenitalen lobären Emphysem kommt es durch einen Ventilmechanismus zu einer Überblähung eines Lungenlappens, bei Progredienz dieses Befundes zu einer lebensbedrohlichen Mediastinalverschiebung. Durch Flüssigkeitsretention kann der betroffene Lungenlappen im ThoraxBild zunächst als Verschattung imponieren, erst im Verlauf tritt die Überblähung als Transparenzvermehrung in Erscheinung. Bei der CCAM ist das Lungengewebe von multiplen Zysten durchsetzt; sie betrifft meist nur einen Lungenlappen und manifestiert sich vor allem auf der linken Seite. Nach Stocker werden bei CCAM 5 Typen unterschieden, Typ 1 und 2 sind häufige Varianten. Auch hier sind die Zysten als Verschattung oder als Transparenzvermehrung abzugrenzen.

Bei der pulmonalen Sequestration werden intra- und extralobäre Typen unterschieden. Diese Läsionen bestehen aus funktionslosem Lungengewebe, das keinen Anschluss an das Bronchialsystem besitzt und von einer Systemarterie versorgt wird. Oft finden sich innerhalb der Sequestration wie bei der CCAM Zysten im Sinne einer „Hybrid-Läsion“. In der neonatalen Thorax-Aufnahme stellt sich diese Fehlbildung meist als Verschattung im Unterlappen dar. Die intralobären Formen werden im Verlauf strahlentransparent, bei extralobären Typen persistiert die Verschattung durch fehlenden Bronchialanschluss. Die arterielle Versorgung aus der Aorta kann mit der Dopplersonografie, der MRT oder der CT dargestellt werden.
Lungenerkrankungen, die bei Neugeborenen fokal oder multifokal imponieren können, sind die neonatale Pneumonie, das interstitielle pulmonale Emphysem (PIE) und die Aspirationspneumonie. Bei der neonatalen Pneumonie kommt es prä, peri- oder postnatal zur Infektion, häufig durch B-Streptokokken, die bei vaginaler Geburt von der Mutter auf das Kind übertragen werden. Im Röntgenbild lassen sich fokal oder diffus angeordnete, fleckförmig bis feinnoduläre Infiltrate abgrenzen; in manchen Fällen fällt die Abgrenzung zu einem Atemnotsyndrom, einer Mekoniumaspiration oder einer transitorischen Tachypnoe schwer. Hier müssen Laborbefunde und der klinische Verlauf ergänzend zur Diagnosestellung herangezogen werden.

Das PIE tritt häufig bei kontrolliert beatmeten Frühgeborenen mit einem Atemnotsyndrom auf; es kann ein- oder beidseitig, fokal oder multifokal sein. Komplizierend kann ein Pneumothorax, ein Pneumomediastinum oder ein Pneumoperitoneum auftreten. In der Thorax-Aufnahme lassen sich beim PIE multiple, winzige, zystoide Aufhellungen bis in die Lungenperipherie abgrenzen. Diese Veränderungen entsprechen kleinsten interstitiellen Luftansammlungen. Das PIE entsteht meist akut in der ersten Lebenswoche und grenzt sich so gegen die sich langsam entwickelnden chronischen Lungenerkrankungen ab.

Zu einer Aspirationspneumonie kann es bei Schluckstörungen oder tracheoösphagealen Fisteln im Rahmen einer Ösophagusatresie oder einer Bronchusfehlbildung kommen. Diese Verschattungen entwickeln sich kurz nach Fütterung. An einen aberrierenden, aus dem Ösophagus abgehenden Bronchus ist zu denken, eine Atresie des Ösophagus kann durch Legen einer Magensonde und anschließende Röntgenuntersuchung ausgeschlossen werden.

Zusammenfassend ist die Thorax-Aufnahme bei respiratorischen Erkrankungen des Neugeborenen die Untersuchungsmethode der ersten Wahl. Eine eingehende Kenntnis neonataler Krankheitsbilder und ihrer fokalen oder multifokalen Manifestation ist die entscheidende Vorrausetzung zu einer korrekten Diagnosestellung. 
Interessenkonflikt

Die Autoren geben an, dass kein Interessenkonflikt besteht.

Autorinnen/Autoren

Andreas Leenen

Katholisches Kinderkrankenhaus Wilhelmstift

Hamburg, Abteilung für Bildgebende Diagnostik
Bibliografie

Dr. med. Andreas Leenen

Katholisches Kinderkrankenhaus

Wilhelmstift

Abteilung für Bildgebende Diagnostik

Liliencronstr. 130

22149 Hamburg

a.leenen@kkh-wilhelmstift.de
DOI https://doi.org/10.1055/a-0943-1248 Online-Publikation: 2019

Fortschr Röntgenstr 2019; 191: S116-S119

(c) Georg Thieme Verlag KG, Stuttgart · New York ISSN 1433-5972 